\title{
Vitamina D y sus implicaciones en estados de salud y enfermedad del ser humano
}

\author{
Dr. Jorge Mauricio Montero García \\ Microbiólogo Químico Clínico 1719, Hospital Los Chiles, Sección Bacteriología; Teléfono: 8887-3174 / 2245-5779; \\ mont26@hotmail.com
}

Recibido: 23 de febrero, 2017

Aceptado: 31 de marzo, 2017

\begin{abstract}
RESUMEN
La vitamina D tiene un extenso papel en el estado de salud y enfermedad, y está ampliamente relacionada con el metabolismo óseo y el equilibrio fosfocálcico, sin embargo, investigaciones de los últimos años demuestran que esta vitamina juega un papel importante en distintas partes del organismo y tiene un rol transcendental en diversos mecanismos como la autotolerancia inmunológica, control del ciclo celular y apoptosis, función endotelial, secreción y efecto de la insulina, regulación del sistema renina-angiotensina-aldosterona, efectividad del sistema inmune ante infecciones, así como implicaciones en el sistema cardiovascular y el desarrollo neurológico, entre otras acciones.
\end{abstract}

Palabras clave: Vitamina D, metabolismo del calcio, deficiencia, respuesta inmune.

\begin{abstract}
Vitamin D has an extensive role in health and disease, and is largely related to bone metabolism and calcium-phosphorus balance, however, research in recent years show that this vitamin plays an important role in different parts of the body and It has a transcendental role in various mechanisms such as immune self-tolerance, cell cycle control and apoptosis, endothelial function, secretion and effect of insulin, regulation of the renin-angiotensin-aldosterone system, effectiveness of the immune system against infections and implications cardiovascular system and neurological development, among other actions.
\end{abstract}

Key words: Vitamin D, calcium metabolism, deficiency, immune response.

\section{Introducción}

La vitamina D es una compleja pro-hormona con innumerables acciones en múltiples sistemas fisiológicos, lo que explica la diversidad de patologías que están relacionadas a su deficiencia. Esta vitamina en su forma activa funciona como una hormona esteroide que induce respuestas fisiológicas en más de 36 tipos celulares que expresan sus receptores (VDR) (Zuluaga et al, 2011). Esta vitamina es además necesaria para la homeostasis del calcio y el fósforo, aumenta la absorción intestinal de calcio, mantiene la concentración de calcio iónico dentro de límites normales en el líquido extracelular, y actúa a nivel del metabolismo óseo. Su déficit provoca raquitismo en el niño y osteomalacia en el adulto (Estefanell et al, 2012)

Recientemente la deficiencia de vitamina D ha sido identificada como un riesgo potencial de muchas otras enfermedades no tradicionalmente relacionadas con esta sustancia, tal es el caso del cáncer y enfermedades cardiovasculares. Por otro lado, se ha demostrado que niveles bajos de esta vitamina se han asociado con otros trastornos como hipertensión y diabetes mellitus, con marcadores de ateroesclerosis como grosor de la capa íntima-media y calcificación coronaria, así como con eventos tales como infarto del miocardio, derrames y hasta falla cardíaca congestiva (Rodriguez, 2011, González, 2009, Sánchez, 2010).

\section{Metabolismo de la vitamina $D_{3}$}

Hay dos fuentes de vitamina D: la vitamina $\mathrm{D}_{3}$ o colecalciferol que es generada por exposición a luz UV y la vitamina $\mathrm{D}_{2}$ o ergocalciferol que se obtiene a través de los alimentos. La vitamina $\mathrm{D}_{3}$ es una hormona que actúa sinérgicamente con la paratohormona (PTH), una necesita 
de la acción de la otra para cumplir su función neta completa (Holick, 2007, Joshi et al, 2010).

Para tener actividad biológica la vitamina $\mathrm{D}_{3}$ debe sufrir 2 hidroxilaciones, la primera sucede una a nivel de hígado por los hepatocitos catalizada por la 25-hidroxilasa que transforma el colecalciferol en 25- hidroxicolecalciferol o calcidiol y luego este último viaja por plasma unida a la proteína de unión a vitamina $\mathrm{D}$ hasta el riñón donde sufre la segunda hidroxilación por la enzima mitocondrial 1-hidroxilasa que transforma el calcidiol en 1,25-hidroxicalciferol o calcitriol, que es vitamina $\mathrm{D}_{3}$ activa y es la responsable de la mayoría de efectos biológicos (Holick, 2007, Kochupillai, 2008)

Hay otras enzimas a nivel renal que generan otros derivados, como el 24,25-hidroxicalciferol producido por la enzima 24-hidroxilasa, pero no tienen actividad fisiológica y es un producto de desecho. Entonces, cuando hay un exceso de la Vitamina $\mathrm{D}_{3}$ se desvía la síntesis para la generación de 24,25- hidroxicalciferol (Sánchez, 2010).

La función principal de la vitamina $\mathrm{D}$ es mantener la concentración de calcio iónico dentro de límites normales en el líquido extracelular. Para cumplir este papel, la vitamina D estimula la absorción intestinal de calcio y promueve la formación de osteoclastos maduros, los cuales movilizarán calcio desde el hueso al extracelular en la fase de resorción ósea del remodelamiento óseo (González et al, 2009).

Hay múltiples factores que pueden influir en los niveles de vitamina $\mathrm{D}$, algunos de ellos son:

- La exposición solar. Se deben tener en cuenta diversos aspectos además del tiempo de exposición como son: la estación del año (se estima que los niveles de $25(\mathrm{OH}) \mathrm{D}$ descienden un $20 \%$ desde finales de verano a pleno invierno), la latitud (en áreas por encima de los $40^{\circ}$ de latitud, generalmente no se alcanza este umbral en invierno y debe almacenarse suficiente vitamina D durante el resto del año), factores climatológicos como la nubosidad y la contaminación. También influyen la superficie corporal expuesta y la protección solar, por lo que hay que tener en cuenta tanto el uso de cremas solares protectoras como la ropa. La pigmentación de la piel es uno de los factores más importantes, ya que afecta al tiempo necesario para producir vitamina $\mathrm{D}$; así, los individuos de piel oscura necesitan entre 3-4 veces más de exposición solar para alcanzar los mismos niveles de vitamina $\mathrm{D}$ que los de piel clara.

- Las enfermedades asociadas con malabsorción de las grasas como la enfermedad inflamatoria intestinal, la insuficiencia pancreática, la fibrosis quística o la colestasis hepática se asocian con concentraciones séricas bajas de vitamina $\mathrm{D}$. Las enfermedades hepática y renal pueden impedir la adecuada hidroxilación de la vitamina D a sus formas activas y causar déficit de vitamina D.

- La administración de determinados fármacos como los antiepilépticos (ej. fenobarbital, fenitoína), rifampicina, antirretrovirales o corticoides a largo plazo, se ha asociado también a niveles bajos de $25(\mathrm{OH}) \mathrm{D}$.

- Los niños alimentados exclusivamente con lactancia materna y los ancianos que viven en residencias, así como las personas con obesidad, son grupos poblacionales con mayor riesgo de déficit de vitamina $\mathrm{D}$ (Rosen, 2011).

\section{Regulación de la Vitamina D}

La 1,25 dihidroxivitamina $\mathrm{D}_{3}(1,25(\mathrm{OH})$ D3) requiere una estricta regulación de activación y desactivación a través de una serie de procesos de retroalimentación positiva y negativa que resultan en cambios en la expresión de las hidroxilasas con el fin de aumentar o disminuir las concentraciones de 1,25 dihidroxivitamina $\mathrm{D}_{3}$ (Plum et al, 2009).

Niveles bajos de calcio son sensados por receptores sensibles al calcio en la paratiroides, por lo que aumenta la expresión de la PTH la cual induce la transcripción de la $1 \alpha$ hidroxilasa en las células tubulares del riñón por lo que se incrementa la producción de la 1,25 dihidroxivitamina $\mathrm{D}_{3}$. Por lo que para regular este ciclo la misma 1,25 $(\mathrm{OH}) \mathrm{D}_{3}$ suprime la producción de PTH y regula negativamente la producción 
de $1 \alpha$ hidroxilasa (Dusso et al, 2005). Asi mismo la 1,25 $(\mathrm{OH}) \mathrm{D}_{3}$ induce la producción de 24 hidroxilasa la cual esta inhibida por los niveles bajos de calcio y por la PTH, esta hidroxilasa origina una autorregulación para atenuar a la $1,25(\mathrm{OH}) \mathrm{D}_{3}$ para protegerse de una hipercalcemia. Además de la PTH, los estrógenos, la insulina, la calcitonia, la prolactina y el factor de crecimiento ejercen una regulación endocrina sobre la actividad de la $1 \alpha$ hidroxilasa, su efecto estimula la acción de esta enzima y favorece la acción de la 1,25 (OH) $\mathrm{D}_{3}$ (Zuluaga et al, 2011)

Dentro de los efectos principales está promover la absorción intestinal de calcio y fosforo y liberar calcio y fosfato de la matriz mineral ósea. Una vez corregido el déficit de calcio sérico se genera una regulación a la baja del eje 1,25 $(\mathrm{OH})$ D3 - PTH, el cual es controlado por el factor de crecimiento fibroblastico-23 (FGF-23), liberado a partir del hueso (St-Arnaud, 2009).

\section{Efectos de la Vitamina $D$ sobre el intestino}

La absorción intestinal de calcio ocurre principalmente en el duodeno. La hormona se vale de la calbindina (Brandan et al, 2012) la cual promueve la absorción de calcio por difusión facilitada, el ligamiento del calcio en el borde en cepillo o superficie luminal (que se incorpora por canales de calcio o por transportadores) y traslado del complejo calbindina-Ca a la membrana basal donde transfiere el ion a una bomba Ca-ATPasa que lo vuelve a la circulación.

La vía no genómica modula un transporte rápido de calcio llamado "transcaltaquia" que involucra la apertura de canales de calcio operados por el voltaje a través de la membrana y transporte vesicular de calcio asociado a la polimerización de microtúbulos que marcan el camino tráfico entre membranas (Zuluaga et al, 2011). El efecto de la vitamina D es fundamental ya que sin la adecuada cantidad de la misma, solo se absorvería $10-15 \%$ del calcio y el $60 \%$ del fósforo ingerido en la dieta (Holick, 2007).

\section{Efectos Óseos}

La calidad ósea mejora gracias a la vitamina $\mathrm{D}$, por los siguientes mecanismos: la absorción intestinal es de un $30 \%$ y puede llegar de un $60 \%$ a $80 \%$ durante periodos de crecimiento activo, aumenta la formación de hueso cortical, disminuye la porosidad cortical, aumenta el número y la función de osteoblastos a través de la inducción de factores de crecimiento óseo y de la síntesis de proteínas de la matriz ósea. Además, aumenta la resistencia a las fracturas y favorece la reparación de microfracturas, ya que sostiene la vitalidad y la función de los osteocitos (Holick, 2007).

Otras células que reciben el mensaje de la hormona $\mathrm{D}$ son los condrocitos, y es posible que entre las funciones de éstos esté controlar la actividad de los osteoclastos: los condrocitos estimulados por el calcitriol sintetizan RANKL, y éste estimula la osteoclastogénesis. La señalización a través del RVD en condrocitos es importante para la función de los osteoclastos y para una correcta homeostasis del fósforo, se ha observado que la carencia de vitamina D disminuye grandemente la función de los osteoblastos. Clásicamente se acepta que la hipovitaminosis D impide la normal mineralización del osteoide y lleva a una severa enfermedad ósea, la osteomalacia o el raquitismo (Sanchez, 2010).

\section{Efectos en Glándula Paratiroides}

La función endocrina de la 1,25 - hidroxivitamina $\mathrm{D}_{3}$ ejerce un potente efecto modulador sobre la función de las glándulas paratiroides. Cuando existe deficiencia de vitamina D se produce hiperplasia de las paratiroides e incremento en la síntesis y secreción de la PTH. La administración de vitamina $\mathrm{D}$ inhibe la síntesis de la PTH y el crecimiento de las células paratiroides, por lo que es una terapia efectiva para el hiperparatiroidismo secundario a enfermedad renal crónica (Norman, 2008). 


\section{Efectos en Riñón}

El efecto más importante de la vitamina $\mathrm{D}$ es la regulación de su propia homeostasis a través de la supresión de la 1-hidroxilasa y de la inducción de la 24-hidroxilasa. Además aumenta la reabsorción tubular de calcio, la expresión de calbindina y acelera el transporte de calcio en el túbulo distal inducido por la PTH (Zuluaga et al, 2011).

\section{Vitamina D y Sistema Inmune}

La vitamina D favorece el control de las infecciones, de las enfermedades autoinmunes y el rechazo a los trasplantes por un ambiente tolerogénico. Estas acciones se explican por el efecto sobre la diferenciación y modulación de la respuesta de monocitos-macrofafos, las células presentadoras de antígenos, las células dendríticas y los linfocitos (Dusso, 2005).

El VDR se encuentra presente en las células presentadoras de antígenos (macrófagos y células dendríticas) y células $\mathrm{T}$ activadas. Macrófagos activados y las células dendríticas son capaces de secretar y sintetizar $1,25(\mathrm{OH}) \mathrm{D}_{3}$ ya que poseen $1 \alpha$ hidroxilasa. Cuando los valores de vitamina D son inadecuados, los macrófagos sólo son capaces de producir cantidades limitadas de 1,25 (OH) D3. Ello explicaría, por ejemplo, el deterioro de la función y activación de los macrófagos asociado a una alta prevalencia de infecciones en los niños con raquitismo (González et al, 2009).

Además, en presencia de 1,25 $(\mathrm{OH}) \mathrm{D}_{3}$ las células dendríticas maduras tienen menos expresión de las moléculas del complejo mayor de histocompatibilidad (MHC) clase II y de moléculas de adhesión necesarias para la estimulación de células T; además inhibe la interleuquina 12 (IL12), la citoquina más importante en el reclutamiento y activación de las células $\mathrm{T}$ hacia a una respuesta Th1, a través de la interferencia con el factor nuclear k B (FN-kB), inhibe a otras citoquinas de las células T, como IL2 y el interferón $\gamma$ (IFN $\gamma$ ), y estimula algunas citoquinas de respuesta Th2 como la IL 4. De esta manera, la acción global de la vitamina D sobre las células
$\mathrm{T}$, estimulada por citoquinas, podría ser la de dirigir su respuesta hacia una respuesta del tipo Th-2 sobre Th-1, convirtiéndose así en agente de prevención y tratamiento de enfermedades autoinmunes mediadas por Th-1 (González et al, 2009). De esta manera promueve un fenotipo tolerogénico, tal como se ha demostrado en los estudios de trasplante de células $\beta$ pancreáticas, cuyos resultados demuestran una tasa menor de rechazo en los ratones tratados con 1,25 $(\mathrm{OH}) \mathrm{D}_{3}$ (Hewison, 2010).

Algunas de estas acciones autoinmunes ya han sido demostradas en enfermedades como la psoriasis, esclerosis múltiple y diabetes mellitus tipo 1 y se está estudiando su acción en otras patologías autoinmunes como lupus eritematoso sistémico, enfermedad inflamatoria intestinal y artritis reumatoide (González et al, 2009).

Estudios epidemiológicos sugieren una correlación entre las concentraciones de la Vitamina D y la incidencia de infecciones respiratorias incluyendo la influenza (Sabetta et al, 2010). Un estudio llevado a cabo en Costa Rica con una muestra de 1024 niños entre edades de 7-10 años demostraron una asociación entre niveles bajos de vitamina $\mathrm{D}$ y el incremento en marcadores de severidad del asma. El epitelio que recubre las vías respiratorias contiene altos niveles de la enzima que convierte la $25-\mathrm{OH}-\mathrm{D}_{3}$ a su forma activa $1,25-\mathrm{OH}-\mathrm{D}_{3}$. Esta forma activa tiene efectos locales en respuesta a infecciones y puede moderar la respuesta inflamatoria como consecuencia de una infección, además de ello, la vitamina $\mathrm{D}$ tiene efectos benéficos en la respuesta inmune adaptativa a través de efectos en el tipo de respuesta inmune, ya sea Th1 o Th2, mecanismos por los cuales esta vitamina ejerce un rol terapéutico reduciendo las exacerbaciones asmáticas (Brehm et al, 2010)

La vitamina $\mathrm{D}$ tiene la capacidad de inducir la expresión de catelicidina un péptido antimicrobiano en bronquios, queratinocitos y diferentes células epiteliales incluyendo las gastrointestinales y del tracto genitourinario, de tal manera que el daño local o la infección puede estimular la expresión de esta proteína en la mayoría de los epitelios, con efectos de inmunidad 
innata y efectos antimicrobianos en contra de bacterias, virus y hongos (Zuluaga et al, 2011).

\section{Vitamina D y el cáncer}

El interés en la vitamina D como un factor protector del cáncer comenzó en 1980 cuando Garlan et al realizaron el mapa de mortalidad por cáncer en EE.UU. y vieron que el cáncer de colon era menor en el sur del país. Se planteó así la posible asociación entre la exposición al sol y un menor riesgo para este tipo de cáncer. Luego se demostró una asociación similar para el cáncer de mama, ovario, linfoma no-Hodgkin y próstata. Apoyando esta asociación se ha demostrado que los grupos con mayor riesgo de deficiencia de vitamina $\mathrm{D}$ presentan peor pronóstico de mortalidad asociado al cáncer, por ejemplo, la obesidad, patología en la que se observan niveles más bajos de vitamina $\mathrm{D}$ que en población eutrófica, presenta una mayor mortalidad para diferentes tipos de cáncer, como colon, mama y próstata (González et al, 2009)

El primer indicio que sugirió la relación de la vitamina $\mathrm{D}$ con el cáncer fue cuando se observó que células leucémicas tanto murinas como humanas expresaban el receptor RVD y al ser expuestas a la 1,25-dihidroxivitamina $\mathrm{D}$ se reducía su actividad proliferativa y se diferenciaban a macrófagos de apariencia normal. Además estudios in vitro han encontrado que la exposición de células tumorales a altas concentraciones de vitamina $\mathrm{D}$ inhibe su proliferación frenando el ciclo celular en fase $G_{0} / G_{1}$ y en algunos casos induce su diferenciación a células más maduras y menos malignas, además tiene un efecto proapoptótica principalmente en células tumorales de cáncer de próstata y mama causando daño en la función mitocondrial con liberación de citocromos y especies reactivas de oxígeno, inhibición y metástasis, efecto anti inflamatorio e inhibición de la angiogénesis actuando sobre las células endoteliales en un microambiente tumoral (Zuluaga et al, 2010)

\section{Vitamina $D$ y enfermedades cardiovasculares}

La mayor información del rol protector de la vitamina $\mathrm{D}$ en la enfermedad cardiovascular (ECV) proviene de los pacientes con insuficiencia renal crónica terminal (IRC). Los pacientes con IRC bajo hemodiálisis o peritoneodiálisis presentan una mortalidad ajustada por ECV que es 10 a 20 veces más alta que la población general. Así mismo, se ha visto que el uso de vitamina $\mathrm{D}$ o análogos de vitamina $\mathrm{D}$ se asocia a reducción del riesgo de muerte por ECV. Por otro lado, es bien conocido que el aumento de los niveles de PTH incrementa la presión arterial y la contractilidad miocárdica, llevando a la hipertrofia de los cardiomiocitos y a fibrosis intersticial del corazón. Por lo tanto, el exceso de PTH contribuye a la ECV (González et al, 2009).

La vitamina D podría influir también en la ECV dado que participa en la regulación de la presión arterial influenciando la activación del Sistema Renina Angitensina (SRA). El calcitriol es un regulador negativo del SRA. En algunos casos el tratamiento con calcitriol mostró una reducción en la actividad de la renina, niveles de angiotensina II, presión sanguínea e hipertrofia miocárdica (González et al, 2009).

\section{Vitamina D y la Diabetes Mellitus}

En mujeres con diabetes tipo 2, la prevalencia de deficiencia de Vitamina D es tres veces mayor que un grupo control (Oh et al, 2009), se ha reportado que la mejoría en el estatus de vitamina $\mathrm{D}$ en pacientes diabéticos tipo 2 mejora la resistencia a la insulina. Esto se explica porque las células beta del páncreas expresan el VDR y su estimulo por parte de la 1,25-hidroxivitamina $\mathrm{D}$ facilita la producción de insulina, adicionalmente el VDR se expresa en los tejidos blanco de la insulina favoreciendo la sensibilidad de estos tejidos a dicha hormona (Zuluaga et al, 2010). 


\section{Niveles de Vitamina D}

El nivel de $25(\mathrm{OH}) \mathrm{D}$ es el mejor indicador del estado global de vitamina $\mathrm{D}$ ya que refleja el total de vitamina $\mathrm{D}$ obtenido tanto de la dieta como de la exposición solar y de la conversión de los depósitos adiposos del hígado. Sin embargo, existe controversia sobre las concentraciones séricas de $25(\mathrm{OH}) \mathrm{D}$ que se asocian a déficit, a salud ósea adecuada y a estado de salud óptimo y los niveles de corte no se han desarrollado por un proceso de consenso específico. Muchas publicaciones recientes consideran el nivel establecido por consenso de $30 \mathrm{ng} / \mathrm{ml}$ como nivel de corte para establecer nivel suficiente, y valores entre 21 y $29 \mathrm{ng} / \mathrm{ml}$ de $25(\mathrm{OH}) \mathrm{D}$ como déficit relativo o insuficiencia, valores por debajo de 20 $\mathrm{ng} / \mathrm{mL}$ se consideran como déficits y por encima de $150 \mathrm{ng} / \mathrm{mL}$ llegan a niveles de intoxicación.

Hay varias moléculas que se pueden cuantificar y que tienen interés clínico, una de ellas es 1,25 (OH) D o calcitriol que es la forma biológicamente activa, tiene una vida media corta de 3-5 días, pero su producción está alterada en insuficiencia renal y tiene concentraciones picomolares. La otra molécula es el 25 hidroxivitamina D o calcidiol, esta presenta una vida media más larga de alrededor de 21 días, tiene concentraciones nanomolares, y es el índice más fiable del estatus global de la vitamina D.

Los métodos de referencia son HPLC y Cromatografía liquida con espectrometría de masas en tándem. Sin embargo se hacen determinaciones con RIA, ELISA o inmunoensayos quimioluminiscentes.

\section{Conclusiones}

La vitamina D tiene múltiples implicaciones en la salud del ser humano, su deficiencia se constituye un riesgo para una gran variedad de enfermedades por lo que la vuelve fundamental para muchos procesos fisiológicos. La deficiencia de esta vitamina es mucho más frecuente de lo que se piensa, sin embargo, el análisis de ésta no está dentro de los exámenes de rutina en nuestro sistema de salud pública. Un mayor conocimiento de las acciones de la vitamina $\mathrm{D}$ podría guiar hacia nuevos tratamientos con el fin de controlar enfermedades de alta prevalencia las cuales son bastante onerosas para cualquier sistema de salud.

\section{Bibliografía}

BRANDAN, N., LLANOS, I. \& RODRIGUEZ, A. (2012). Regulación hormonal del balance fosfocálcico. Universidad Nacional del Nordeste. Facultad de Medicina. Cátedra de Bioquímica.

BREHM, J., SCHUEMANN, B., FUHLBRIGGE, A., HOLLIS, B., STRUNK, R., ZEIGER, R., WEISS, S. \& LITONJUA, A. (2010). Serum vitamin D levels and severe asthma exarcebations in the childhood asthma management program study. Journal Allergy Clinical Immunology. 126(1): 52-58.e5. Doi: 10.1016.

DUSSO, A., BROWN, A. \& SLATOPLOSKY, E. (2005). Vitamin D. American Journal of Physiology Renal. 289, 8-28.

ESTEFENELL, C., OLIVERA, R., SATRIANO, R. \& TANZI, M. (2012). Pauta Vitamina D. Archivos de Pediatría de Uruguay. 83(1), 24-27.

GONZÁlEZ, G. \& TORREJON, C. (2009). Actualizaciones en Vitamina D: Revista Chilena de Reumatología. 25(2), 83-87.

HEWISON, M. (2010). Vitamin D and Immune System: New Perspectives on an Old Theme. Endocrinology Metabolism Clinics of North America. 39, 365-379.

HOLICK, M. (2007). Vitamin D Deficiency. New England Journal of Medicine, 357, 266-81.

HOLICK, M. (2011). Health Benefits of Vitamin D and Sunlight: a D-bate. Nature Endocrinology. 7, 73-75

JOSHI, D., CENTER, J., \& EISMAM, J. (2010). Vitamin $\mathrm{D}$ deficiency in adults. Australian Prescriber. 33(4): 103-106.

KOCHUPILLAI, N. (2008). The Physiology of Vitamin D: current concepts. Indian Journal Medical Research. 127, 256-262.

NORMAN, A. (2008). From vitamin D to hormone D: fundamentals of the vitamin D endocrine system essential for good health. American Journal of Clinical Nutrition, 88: 491S-499S. 
OH, J., WENG, S., FELTON, S., BHANDARE. S., RIEK, A., BUTLER, B., PROCTOR, B., PETTY, M., CHEN, Z., SCHECHTMAN, K., BERNAL-MIZRACHI, L. \& BERNAL-MIZRACHI, C. (2009). 1,25(OH) Vitamin D inhibits foam cell formation and suppresses macrophage cholesterol uptake in patients with type 2 diabetes. National Institutes of Health. 120(8): 687-698. doi: 10.1161.

PLUM, L. \& DELUCA, H. (2009). The Functional Metabolism and Molecular Biology of Vitamin D Action. Clinical Reviews in Bone and Mineral Metabolism 7, 20-41.

ROSEN, C. (2011). Vitamin D Insufficiency. New England Journal of Medicine 364. 248-54.
SABETTA, J., DEPETRILLO, P., CIPRIANI, R., SMARDIN, J., BURNS, L. \& LANDRY, M. (2010). Serum 25-Hydroxyvitamin D and the incidence of acute viral respiratory tract infections in healthy adults. Plos One. 5, Issue 6, e1 1088.

SÁNCHEZ, A. (2010). Vitamina D: Actualizacion. Revista Médica de Rosario. 76. 70-87.

ST-ARNAUD, R. (2009). The Direct Rol of Vitamin D on Bone Homeostasis. Archives Biochemestry Biophysics. 473, 225-230.

ZULUAGA, N., ALFARO, J., GONZÁLEZ, V., JIMÉNEZ, K. \& CAMPUZANO, G. (2011). Vitamina D: Nuevos Paradigmas. Medicina y laboratorio 17(5) 211-246. 
